\title{
The SPIDERobot: A Cable-Robot System for On-Site Construction in Architecture
}

\author{
José Pedro Sousa ${ }^{1}$, Cristina Gassó Palop ${ }^{1}$, Eduardo Moreira ${ }^{2}$, Andry Maykol Pinto², \\ José Lima ${ }^{3}$, Paulo Costa ${ }^{4}$, Pedro Costa ${ }^{4}$, Germano Veiga ${ }^{2}$, A. Paulo Moreira ${ }^{4}$ \\ ${ }^{1}$ Faculty of Architecture, University of Porto + CEAU/DFL \\ \{jsousa, cpalop\}@arq.up.pt \\ 2 INESC TEC \\ eduardo.j.moreira@inesctec.pt, andry.pinto@fe.up.pt, \\ germano.veiga@inescporto.pt \\ ${ }^{3}$ INESC TEC + Polytechnic Institute of Bragança \\ jlima@ipb.pt \\ ${ }^{4}$ INESC TEC + Faculty of Engineering, University of Porto \\ \{paco, pedrogc, amoreira\}@fe.up.pt
}

\begin{abstract}
The use of robots in architectural construction has been a research field since the 1980's. Driven by both productive and creative concerns, different systems have been devised based on large-scale robotic structures, mobile robotic units or flying robotic vehicles. By analyzing these approaches and discussing their advantages and limitations, this paper presents an alternative strategy to automate the building construction processes in on-site scenarios. The SPIDERobot is a cable-robot system developed to perform assembly operations, which is driven by a specific Feedback Dynamic Control System (FDCS) based on a vision system. By describing and illustrating this research work, the authors argue about the advantages of this cable robot system to deal with the complexity and the scale of building construction in architecture.
\end{abstract}

Keywords: Cable-Robot, SPIDERobot, Automated Construction, Digital Fabrication, Non-Standard Architecture

\section{INTRODUCTION}

The use of robotic technologies in architectural construction can be traced back to the 1980 's. By then, robotic technologies were employed in Japan to introduce a high level of automation not only in the factory but also in the construction site. One of the first applications to a full-scale building happened in 1991 in Nagoya, when the S.M.A.R.T. (i.e., Shimizu Manufacturing system by Advancing Robotic Technology) was used to build a 20-storey height building for the Juroku Bank. Despite its automation, these systems still required a lot of manual work and standardization ruling the design of the building and its further construction process [Cousineau and Miura 1998] [Kolarevic

adfa, p. 1, 2011.

(C) Springer-Verlag Berlin Heidelberg 2011 
2001]. As a consequence, these on-site robotic systems didn't prove to be satisfactory and lost some importance overtime.

In 2005, Gramazio and Kohler [2008] recovered the interest in robotics with a focus in enhancing design creativity. In a moment when digital tools assist an unprecedented freedom at the design level, it was crucial to find rigorous and flexible manufacturing technologies. Understood as universal machines that could be adapted to perform different kind of fabrication operations by adding customized end-effectors, industrial robots emerged as a promising technology to be investigated. Gramazio and Kohler's work at the Swiss Federal Institute of Technology in Zurich (ETHZ) has showed, for almost a decade now, how industrial robots can be used to fabricate with different materials and explore a wide range of geometric and tectonic conditions. This kind of research has spread to other schools and research groups and motivated the creation in 2010 of a specific Association for Robots in Architecture ${ }^{1}$ by Sigrid Brell-Cokcan and Johannes Braumann.

However, despite the success of such technological transfer, the use of industrial robots still present some limitations regarding the scale of architecture. Due to the limited range of action and movement of the machine, its application seems more optimized to embrace prefabrication logics than on-site construction. Indeed, the precise, controlled and safe environment of the factory space provides the perfect setup to work with industrial robots. On the contrary, the accidental and weather exposed conditions found in on-site construction sets highly unstable conditions to work with such machines. To overcome this situation, the industrial robot must integrate additional technological devices and behaviors, like movable platforms, feedback sensors and cameras, auto-programming techniques for real-time functional adjustment, etc. In short, the adaptation of the industrial robot for on-site construction is complex and so, other robotic approaches may be explored. By considering this move from the fabrication of components to the construction of buildings, the next chapter surveys some of the current trends facing automation and robotics in construction.

\section{ROBOTIC SYSTEMS FOR ON-SITE CONSTRUCTION}

Looking for introducing robotic technologies in the on-site construction, engineers and architects have explored several strategies to deal with the complexity of such challenge. For a better understanding of current tendencies in the field, this paper proposes to consider the following categories:

- Large Scale Robotic Structures

- Mobile Robotic Units

- Flying Robots

1 http://www.robotsinarchitecture.org (accessed in June 2015) 


\subsection{Large Scale Robotic Structures}

This section comprises those approaches looking for augmenting the scale of conventional robotic setups to fit them to the scale of the architectural buildings. The traditional scaffolding of construction sites or the gantry systems of the CNC routers are some of the structures that can be potentially enlarged and automated.

The robotic construction initiatives in the 1980's and 1990's in Japan, like the S.M.A.R.T. system mentioned above, are similar to a big scaffolding structure, which integrates robotic systems at many levels to perform different construction operations. Bock and Langerberg [2014: 98] refer to this concept as Integrated Automated Construction Sites and describe them as "partly automated, vertically moving on-site factories providing shelter for an on-site assembly". The Waseda Construction Robot Group (WACOR) was also one of the first initiatives to promote this trend.

In a different way, Behrokh Koshnevis devised a large-scale gantry bridge structure moving horizontally along two parallel lanes to support his Contour Crafting technology [Koshnevis and Bekey 2002]. At the University of Southern California, the team conceived an automated system for carrying a material deposition nozzle to 3D print architectural buildings in a single-run. Resembling a big CNC router, this type of structure concept has inspired many other similar strategies for on-site additive construction processes, like the D-Shape technology ${ }^{2}$ developed by Enrico Dini.

Despite the robustness and high operational capacities proposed by these systems, their real application in the construction sites is far from being easy. According to Gambao et.al. [1999: 600], "these big and heavy robots are difficult to transport to the construction site, have some unsolved scientific and technical problems, and need a very high investment”.

\subsection{Mobile Robotic Units}

To overcome the stationary condition of industrial robots and cope with the large size of building constructions, the placement of robots over mobile platforms has been another research avenue.

Started in 1992, the ROCCO (Robot Assembly System for Computer Integrated Construction) project developed by a European Consortium of Schools and Research Centers departed from the understanding that an "articulated robot placed over a mobile platform (a lorry, a towable platform or an autonomous mobile robot) results very appropriate for the assembly tasks on a construction site" [Gambao et.al. 1999: 600]. Besides this mobile condition, two robotic arms were specifically developed to expand the payload and range of action of the conventional ones, with the largest of them having the capacity to handle up to $500 \mathrm{Kg}$ and the reach of $8,5 \mathrm{~m}$. The integrated system aimed at responding to the building construction trends in using progressively larger and heavier material blocks (e.g., in concrete and stone). The intention in automating construc-

2 http://www.d-shape.com (accessed in June 2015) 
tion tasks based in repetitive operations had led to other similar initiatives, like the robotic bricklayer S.A.M. (i.e., Semi-Automated Mason) developed by Construction Robotics [Petters and Belden 2014].

In a similar fashion but with a deeper interest in addressing creative design issues rather than productive efficiency ones, Gramazio and Kohler initiated in 2011 a research line on In Situ Robotic Fabrication at the ETHZ. With such goal, they devised a mobile unit equipped mounted with a robotic arm on the top to perform a variety of construction tasks. In order to assure its adaptation to the "continuous changing conditions, unpredictable events, obstacles, and the activities and movements of people working on-site", the unit integrated additional systems, like sensor and scanning technologies and different end-effectors [Helm et.al. 2012, 169]. The Endless Wall, the Stratifications and the Fragile Structure installations are some of the experiments developed to test such purposes.

The strategy of introducing mobile possibilities to flexible industrial robots is interesting because it avoids the complex setup of heavy large-scale structures. Even when facing the problem of the building height, Coop Himmelb(l)au imagined the use of lifting platforms for the vertical mobility of industrial robots, in a recent proposal presented in a video called "We Start The Future of Construction"3,. Although this last reference is still a vision, the examples based in ground mobility still have to solve some technological problems to overcome the unstructured constraints of the on-site construction environments.

\subsection{Flying Robotic vehicles}

The exploration of aerial modes of robotic construction became the most recent research field for on-site construction. Launched by Gramazio and Kohler in collaboration with Raffaello D'Andrea at the ETHZ in 2011, this idea consisted in using flying vehicles to manipulate building components in the air. In this way, constrains inherent to ground-based mobility and the need for scaffolding or cranes could be avoided [Wilmann et.al. 2012]. Furthermore, this approach can involve the cooperation of several aerial robotic units to allow the execution of different and synchronized building construction tasks.

This team first demonstrated this approached in the Flight Assembled Architecture installation at the FRAC Centre in Órleans (France). A set of four quadcopters lifted, transported and assembled a tower structure made out of 1500 lightweight foam modules (Wilmann et.al. 2012). The research on Aerial Constructions continued in other experiments, by testing the assembly of space frame structures and also the erection of tensile structures (Mirjan et.al. 2014).

As described above, the advantages of the aerial robotic construction applications promise an unprecedented freedom in building construction. As a consequence, this approach can stimulate new ways of thinking and designing architecture, as the student works developed in the FCL design studio in Singapore can demonstrate [Budig et.al

3 http://www.coop-himmelblau.at/architecture/news/we-start-the-future-of-construction (accessed on June 2015) 
2014]. However, this research field is still on an early stage of development. The automation and cooperative control technologies, the energy autonomy or the payload capabilities are some of the important technology challenges to face in the near future.

\section{THE CABLE-ROBOT SYSTEM - SPIDERobot}

Facing this trend on developing automated and flexible modes of on-site construction, this paper presents the research of an alternative technology based on a cable-driven robot system (i.e., referred in this paper as cable-robots), which is called as SPIDERobot. Under development since 2013 at the INESC TEC and the Faculty of Architecture of the University of Porto, this approach is based on similar systems developed in other fields, like the sports and entertainment industries. The Skycam ${ }^{4}$ and the CableCam $^{5}$ are just two examples of cable-cameras that can operate and move throughout the whole three-dimensional space where an event is occurring, like a stadium or a pavilion. The transfer and adaptation of such systems to the field of building construction has the potential to overcome some of the problems found in the examples mentioned in the previous chapter. The next sections describe the principles of the SPIDERobot, as well as the first functional prototype that was built and tested to evaluate the concepts. This research is still on an early stage and the first results are discussed in the Conclusion chapter.

\subsection{System Description}

Cable-driven robots are automated systems where multiple cables are attached to a mobile platform or end-effector. A positioning system controls the cables by actuating the motors for extending or retracting the cables [Bosscher et.al. 2007]. The overall system is thus relatively simple, which opens some interesting advantages to use cable-robots for on-site construction applications.

When compared with other robotic construction systems like those presented in chapter 2, cable-robots are easy and inexpensive to transport, assembly and disassembly in the construction site, due to the lightness of the cable-based system. Furthermore, the configuration of the cable-based structure allows the definition of larger translational working spaces, which is decisive to face the scale of architectural constructions. Unlike flying robotic vehicles, cable-robots can have much higher payloads and work continuously by means of constant energy supply (i.e., avoiding the use of batteries), while complying with the safety requirements. Despite these advantages, cable-robots also present some critical features. The number and movement of the cables can cause interference within the working space, and their force in the downward direction is limited. The cable system also faces specific technological challenges regarding the control of the precision due to the tension forces and some elasticity of the cables.

4 http://www.skycam.tv (accessed on June 2015)

5 http://www.cablecam.com (accessed on June 2015) 
In this context, the SPIDERobot is a low-cost prototype of a cable-driven robot develop to perform assembly operations in on-site construction scenarios. Its structure consists in 4 actuated cables, which are fixed on the top corners of a frame with 120x60x135cm, and connected to a central mobile platform equipped with a rotating gripper (Figure 1). The system configuration presents 4 degrees of freedom (DOF), which comprise the XYZ movements and the rotation angle around $\mathrm{Z}$.
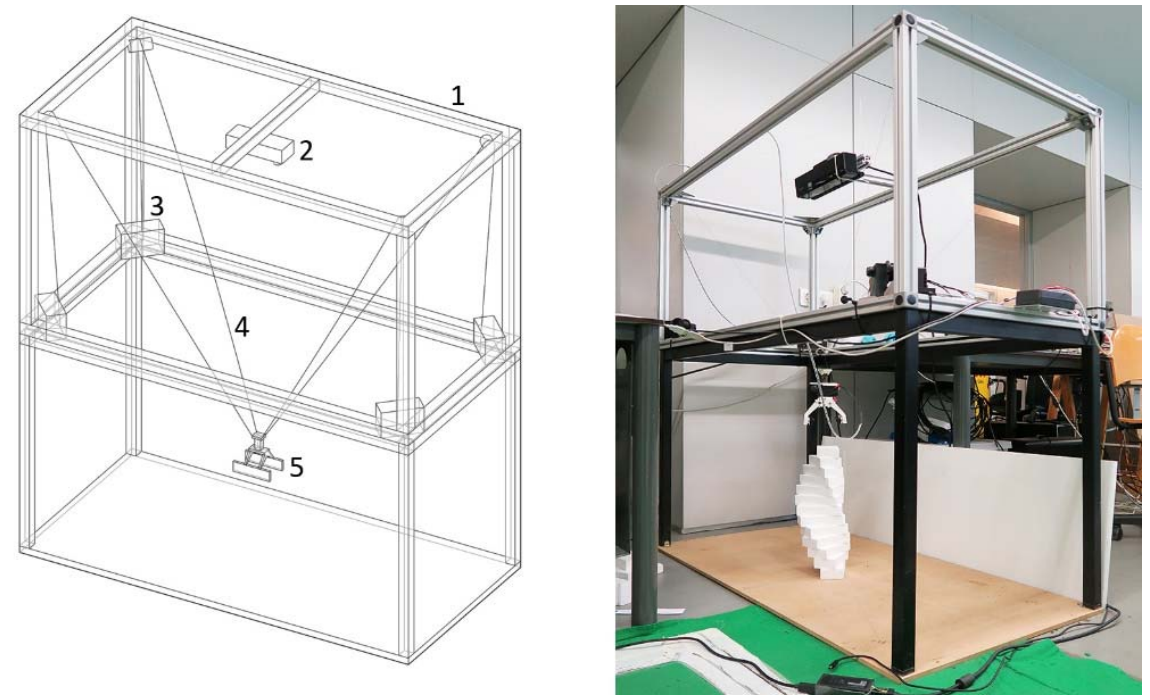

Fig. 1. The description of the SPIDERobot system: (1) frame; (2) vision system; (3) motors; (4) cables; (5) mobile platform with the gripper. On the right, a photo of the built prototype.

Regarding other cable-robot systems, the SPIDERobot presents some combined distinct features. By using only 4 cables, it reduces the possibility for cable interference with obstacles and leaves more useful working space than systems with more cables, like the contour crafting robot system with 12 cables presented in [Bosscher et.al. 2007]. However, because this option leaves the kinematics of the robot under-constrained, the gravity force affects the cables tension and consequently the precision of the whole system [Moreira et.al. 2015]. To deal with this situation, the large majority of cable robots use tensor-feasible controlling systems for positioning the mobile robot in the workspace. In a different way, the SPIDERobot presents a specific Feedback Dynamic Control System (FDCS) that does not require sensors for measuring the cables tension. Instead, the proposed FDCS control is based on a vision-based system, which can be something similar to a differential GPS or laser measurement system on the construction site. By using the information available in the environment, the FDCS controls the positioning of the robot while assuring that the length of the cables is always within safe values. 


\subsection{Practical Experiment}

The SPIDERobot prototype was tested in the assembly of an irregular structure made out of 18 foam blocks with $120 \times 60 \times 30 \mathrm{~cm}$. The design of the structure was modeled in Rhinoceros with the goal of defining a geometry that could challenge conventional modes of construction. Then, the different spatial coordinates and orientation of each block (i.e., defined by the coordinates of two points) were listed in an Excel file with the help of Grasshopper. This information was used to inform the SPIDERobot about the position of the blocks in the structure (Figure 2).

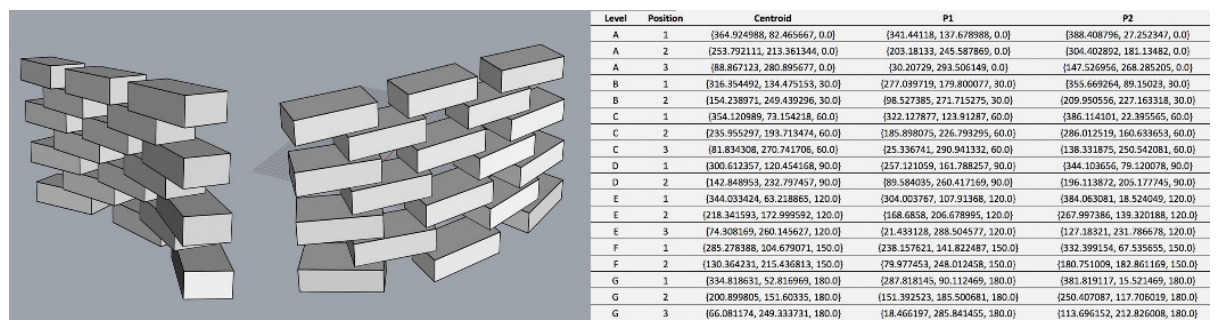

Fig. 2. The design model of the structure used in the experiment (left). The spatial positioning information of the blocks (right).

For picking them from the feeder site, the SPIDERobot took advantage of its FDCS based on a vision system to automatically detect and recognize them in the working space. With this feedback, the robot adjusted its height and orientation to pick the blocks correctly (Figure 3). With this kind of intelligent behavior, the placement of the blocks in the construction feeder site does not have to be rigorous. In the experiment, the blocks were placed in the feeder site in stacks up to 5 units.

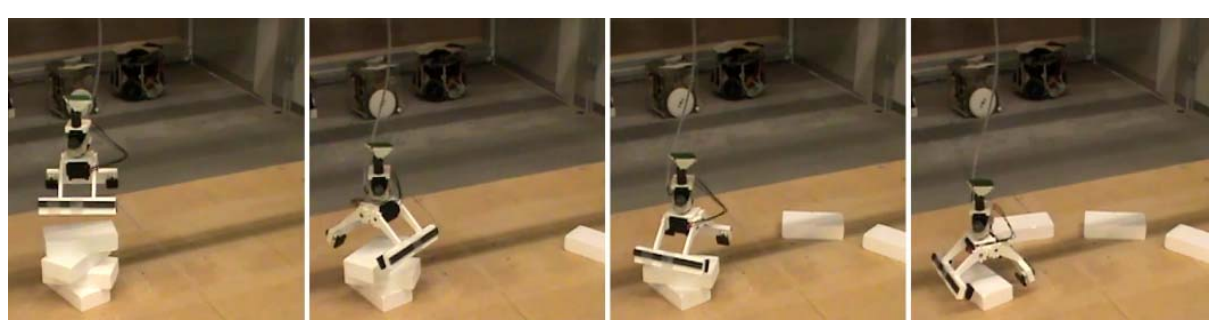

Fig. 3. Sequence showing the automatic rotation of the gripper with the help of the FDCS, when picking the blocks from the feeder site.

The assembly of the 18 blocks was completed in around 16 minutes. The whole process was slow, but revealed to be accurate (Figure 4). 


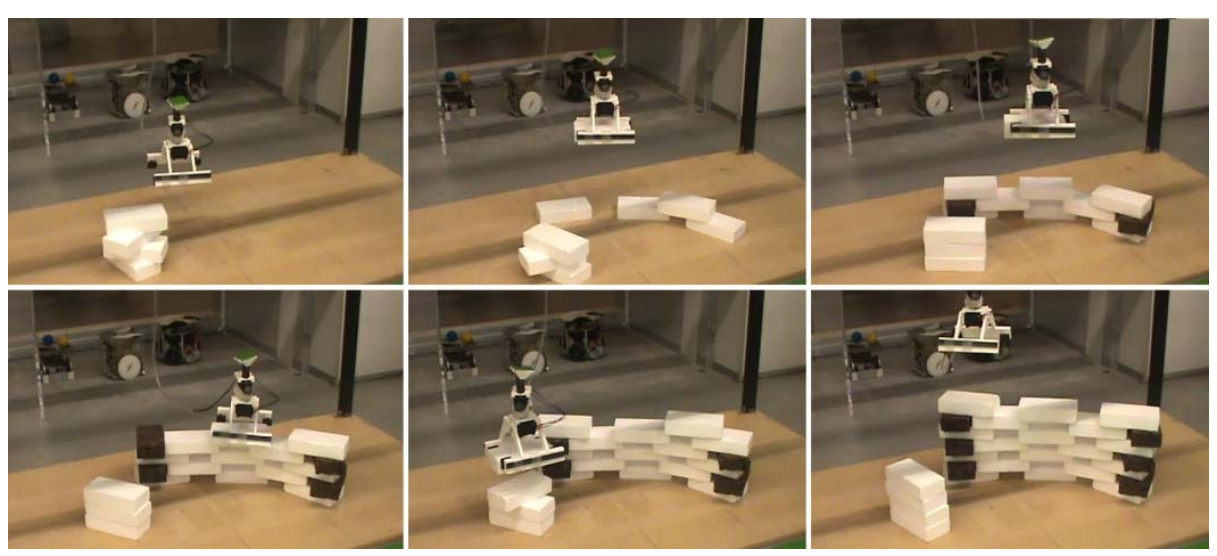

Fig. 4. Sequence of photos showing the SPIDERobot assembling the structure.

\section{CONCLUSION}

This paper presented a cable-robot system as an alternative strategy for automating the on-site construction in architecture. Moved by design creativity concerns, the authors tried to overcome some of the technological, physical and economical limitations presented in other research approaches based on large-scale robotic structures, mobile robotic units or flying robotic vehicles. The current stage of the research work converged in the production of the SPIDERobot prototype, and it can open the discussion at two levels: the technological and the architectural.

On the one hand, in analytical studies conducted by the authors [Moreira et.al. 2015], the results demonstrated that the topology of the FDCS implemented in the SPIDERobot revealed to be more accurate than the traditional force-feasible approach to the kinematics of cable-robots when performing pick-and-place operations. Therefore, the FDCS proved to be a promising system to be scaled to the size of real construction environments [Moreira et.al. 2015]. Currently, the research work is already centered in further exploring the vision system of the FDCS to expand the autonomous capabilities of the system, like in the automatic detection and avoidance of obstacles. Future research directions will be focused in scaling-up the prototype, refining the vision-system and improve the robustness and speed of the motors.

On the other hand, the SPIDERobot has the potential to challenge the traditional concepts of designing and building in architecture. By considering its 4 DOF and the geometric configuration of the 4 cables, architects can incorporate such parameters in the creative process to drive design customization possibilities towards aesthetically pleased and functionally efficient buildings. Indeed, unlike other robotic approaches, the cable-robot system can assist not only the prefabrication of building parts (e.g., brick walls) but also, and above all, the on-site construction of buildings through the assembly of those building parts or, even, the stacking of building units (e.g., prefabricated housing modules) (Figure 5). For instance, the kind of solutions imagined for high-rise building design developed in the FCL design studio conducted by Gramazio 
and Kohler in Singapore [Budig et.al. 2011], could benefit from this approach. Furthermore, the simplicity and flexibility of the system also facilitates its integration in both empty construction sites (e.g., with the help of cranes) and in highly dense urban scenarios (e.g., by taking advantage of existing buildings to set up the cable system) (Figure 6).

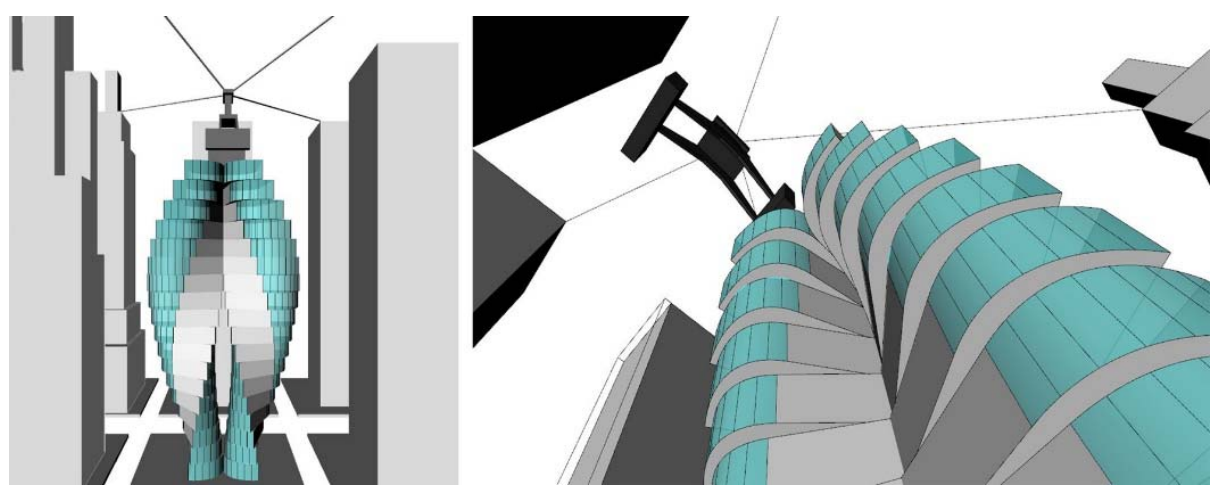

Fig. 5. Envisioning the application of the SPIDERobot system in the construction of novel architectural buildings.
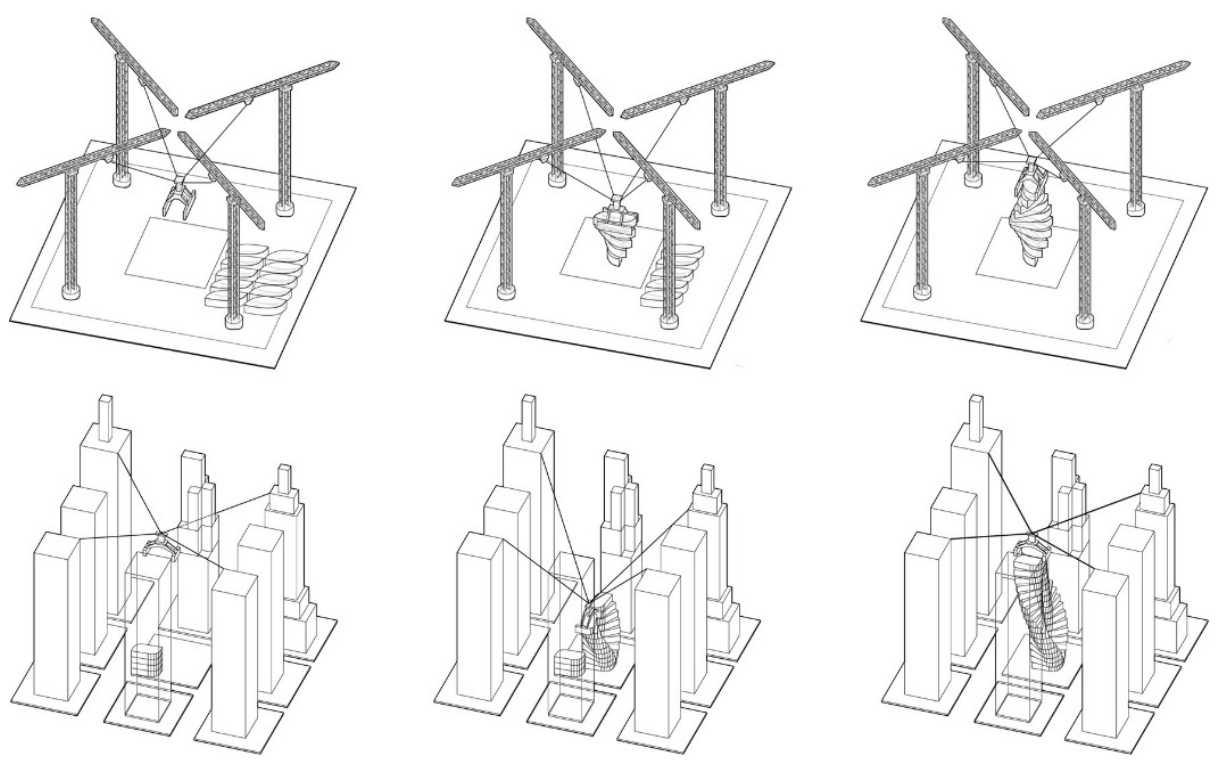

Fig. 6. Envisioning the application of the SPIDERobot system in empty or urban contexts.

In resume, the exploration of cable-driven robots can be an effective solution for stimulating design creativity and expanding digital fabrication processes to the realm of digital construction in architecture. Its application in practice can also foster the vision 
of different and complementary robotic construction technologies cooperating in the on-site construction of architectural buildings.

\section{ACKNOWLEDGMENTS}

This work was developed in the scope of the Research Project with the reference PTDC/ATP-AQI/5124/2012, funded by FEDER funds through the Operational Competitiveness Programme - COMPETE, and by national funds through the FCT - Foundation for the Science and Technology.

\section{REFERENCES}

Bock, T. and Langenberg, S. 2014, “Changing building sites. Industrialization and automation of the building process" in F. Gramazio and M. Kohler (Eds), Made by Robots - Architectural Design n.229, pp. 88-99, John Wiley \& Sons.

Bosscher, P., Williams, R.L., Bryson, L.S., Castro-Lacouture, D. 2007, “Cable-suspended robotic contour crafting system”, in Automation in Construction, Vol.17, Issue 1, p.45-55, May 2008.

Budig, M., Laurer, W.V., Petrovice, R. and Lim, J. 2014, “Design of robotic fabricated high rises” in W. McGee and M. Ponce de Leon (Eds), Robotic Fabrication in Architecture, Art and Design 2014, pp. 111-130, Springer, Michigan USA.

Cousineau, L and Miura, N. 1998, Construction Robots. The Search for New Building Technology in Japan, ASCE Press.

Gambao, E., Balaguer, C. and Gebhart, F. 1999, “A robotic system for automated masonry” in Automation and Robotics in Construction XVI, pp. 509-602.

Gramazio, F. and Kohler, M. 2008, Digital Materiality, Lars Muller Publishers, Basel.

Helm, V., Ercan, S., Gramazio, F., Kohler, M. 2012, "In-situ robotic construction: extending the digital fabrication chain in architecture" in Synthetic Digital Ecologies: Proceedings of the $32^{\text {nd }}$ Annual Conference of the Association for Computer Aided Design in Architecture (ACADIA) 2012, pp. 169-176, San Francisco.

Khoshnevis, B. and Bekey, G. 2002, “Automated construction using contour crafting - applications on earth and beyond”, in Proceedings of the 19th ISARC, pp. 489-495, Washington USA.

Kolarevic, B. 2001, "Digital fabrication: manufacturing architecture in the information age" in W. Jabi (Ed.), Reinventing the Discourse, Proceedings of the ACADIA 2001 Conference, pp. 268-277, Washington DC.

Mirjan, A., Gramazio, F., Kohler, M. 2014, "Building with flying robots” in F. Gramazio, M. Kohler and S. Langenberg (Eds.), FABRICATE: Negotiating Design and Making, pp. 266-271, Zurich.

Moreira, E.; Pinto, A.M.; Costa, P.; Moreira, A.P.; Veiga, G.; Lima, J.; Sousa, J.P.; Costa, P. 2014, "Cable Robot for Non-Standard Architecture and Construction: A Dynamic Positioning System” in Industrial Technology (ICIT), 1015 IEEE International Conference on, pp. 3184-3189.

Petters, S. and Belden, R. 2014, 'SAM, the robotic bricklayer', in SMART / Dynamics of Masonry, 1 (4), pp. 10-14. 
Wilmann, J., Augugliaro, F., Cadalbert, F., D’Andrea, R., Gramazio, F., Kohler, M. 2012,

“Aerial robotic construction towards a new field of architectural research", in International Journal of Architectural Computing Vol. 10-3, pp. 439-459. 